\title{
一种改进量子遗传算法在地空反辐射混编群 兵力配置优化中的应用
}

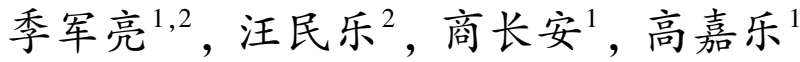

(1.空军工程大学 防空反导学院, 陕西 西安 710051；2. 火箭军工程大学, 陕西西安 710025)

\begin{abstract}
摘 要: 提出了地空反辐射混编群概念及防电磁千扰与抗空中进袭的综合兵力配置需求, 基于电子支 援下的空袭作战背景, 分析了掩护角、配置距离、有效千扰等要素的相互关系, 并以杀伤区面积为目标 函数, 构建了地空反辐射混编群兵力配置优化模型。结合混编群兵力配置特点, 对量子遗传算法进行 了旋转角的自适应改进,在设置想定实例的基础上,运用改进的量子遗传算法对问题进行求解, 并与 传统量子遗传算法、粒子群算法和量子涡流算法计算结果进行了对比分析。结果表明:运用改进量子 遗传算法求解地空反辐射混编群兵力配置优化问题, 全局寻优能力更强、收敛速度更快、稳定性更好。
\end{abstract}

\section{关 键 词: 量子遗传算法;地空反辐射; 混编群; 兵力配置; 量子旋转门 \\ 中图分类号:TJ762.3 3 ; TP391.9 文献标志码: A \\ 文章编号: 1000-2758(2019) 05-0992-08}

地空反辐射导弹 (SA-ARM) 武器系统是利用空 中目标电磁辐射获取探测识别、跟踪制导等信息, 并 对目标实施拦截的地面防空装备, 其主要作用是为 体系内其他信息化作战力量提供电子对抗的 “保护 伞” [1]。由于 SA-ARM 武器系统应对目标类型较为 单一、对空中突击目标防护能力不强等特点, 在防空 作战体系化趋于常态的背景下, 该武器系统与常规 防空装备混编作战就成为必然。以 SA-ARM 武器 系统为主体, 若干常规防空装备为配属组成的防空 集群称为地空反辐射混编群, 在兵力组成既定的前 提下, 常规防空装备如何配置才能发挥地空反辐射 混编群整体火力, 既能实现对空中突防/击目标的有 效拦截, 又能确保 SA-ARM 武器系统对防区外电子 支援飞机的慑阻, 避免电子支援飞机对常规防空装 备的有效干扰, 这已成为混编群作战运用亟待解决 的问题。地空反辐射混编群兵力配置是典型的多约 束决策问题,既要求常规防空装备避免被有效干扰, 又要争取不同型号常规装备混合编组时, 通过合理 的目标分配与火力运用对空袭编队的最大杀伤效能 (杀伤目标数学期望最大化), 围绕该问题相关学者 也进行了探讨, 文献 [2]对地空反辐射导弹的功能
性能特点进行了分析, 提出了其在防空作战体系中 的作用及与常规防空装备混编编配的设想 ${ }^{[2]}$, 文献 [3] 阐述了地空反辐射导弹武器系统与常规防空装 备混编作战的必要性及地空反辐射混成群内涵, 提 出了兵力编组模式和混成群兵力部署的基本要 求 $^{[3]}$ 。总体而言, 目前围绕该问题开展的研究偏 少, 且其中定性论述居多、定量分析缺乏, 可供参考 借鉴的成果不够。量子遗传算法 ( QGA-quantum genetic algorithm) 是传统遗传算法中引人量子计算的 产物, 与传统遗传算法相比, 量子遗传算法具有更好 的种群多样性和并行计算能力, 其搜索范围更广、适 应性更强、计算效率更高 ${ }^{[4]}$ 。本文从地空反辐射混 编群抗击空袭编队在掩护地面目标及反辐射导弹阵 地对空安全、为常规防空装备提供电子防护等作战 需求人手 ${ }^{[3,5]}$, 构建了混编群兵力配置优化模型, 在 提出一种改进的量子遗传算法基础上,将该算法应 用于地空反辐射混编群兵力配置优化问题, 并针对 设定同型号常规防空装备兵力组成的地空反辐射混 编群与作战对象, 直接生成可指导兵力配置的实施 方案, 经仿真对比该算法全局寻优能力更强、收玫速 度更快。 


\section{1 概念描述及关系分析}

为便于开展地空反辐射混编群兵力配置优化分 析,在此引人空袭兵器完成任务线、压制性系数等有 关概念。

\section{1 概念描述}

1) 空袭兵器可能完成任务线

敌空袭兵器可能完成任务线是指敌机或远射导 弹为了使其空袭兵器有效毁伤保卫目标, 所必须到 达保卫目标周围的一条界线, 通常用这一界线到保 卫目标中心的水平距离来表示。如果在此界线前将 空袭目标消灭, 则不会给保卫目标造成伤害, 在此界 线后即使将空袭目标消灭, 仍会给保卫目标造成伤 害 $^{[6]}$, 空袭兵器可能完成任务线如图 1 所示。

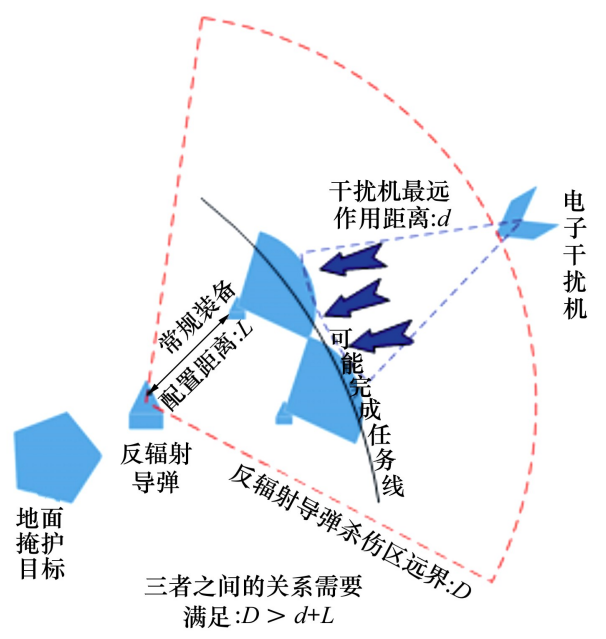

图 1 SA-ARM 杀伤区远界与常规防空装备配置距离 及电子干扰机最远作用距离的关系

2) 压制性系数与有效干扰

电子干扰压制性系数 $K_{j}$ 是指电子干扰设备对 防空武器火控雷达实施有效干扰即在搜索状态下雷 达发现概率下降到 0.1 以下时, 雷达接收机输人端 所需要的最小干扰信号与雷达回波信号功率之比。 从定义来看, 压制性系数越小, 雷达抗干扰能力越 弱, 越容易被干扰; 反之, 压制性系数越大, 雷达抗干 扰能力越强, 越不易被干扰。利用压制性系数可以 推导出干扰方程

$$
\frac{P_{\gamma j}}{P_{\gamma s}}=\frac{P_{j} G_{j}}{P_{t} G_{t}} \times \frac{4 \pi \gamma_{j}}{\sigma} \times \frac{G_{t}^{\prime}}{G_{t}} \times \frac{R_{t}^{4}}{R_{J}^{2}} \geqslant K_{j}
$$

式中, $P_{\gamma s}$ 为雷达收到的目标回波信号, $P_{\gamma j}$ 为进人雷 达接收机的干扰信号, $P_{j}, P_{t}$ 分别为干扰机和雷达的 发射功率, $G_{j}, G_{t}$ 分别为干扰机和雷达的天线增益, $\gamma_{j}$ 为干扰信号对雷达天线的极化系数, $\sigma$ 为目标的 雷达截面积, $G_{t}^{\prime}$ 为雷达天线在干扰机方向上的雷达 天线增益, $R_{t}, R_{J}$ 分别为目标与雷达的距离及干扰 机与雷达的距离 ${ }^{[7-8]}$ 。

\section{3) 掩护角}

防空武器对保卫目标的掩护角, 是一个以保卫 目标中心为顶点的扇面角, 配置在保卫目标周围的 防空武器可以将从该扇面角范围内临近保卫目标飞 行的敌机消灭在其可能完成任务线之前 ${ }^{[9]}$ 。掩护 角 $2 \phi$ 的大小与敌机可能完成任务线到保卫目标中 心的水平距离 $d_{r w x}$ 、杀伤区的远界水平距离 $d_{s y}$ 、防空 武器相对于保卫目标中心的水平距离 $d_{p z}$ 有关。

$$
2 \phi=2 \cos ^{-1} \frac{d_{p z}^{2}+\left(d_{r w x}+0.5 h_{0}\right)^{2}-d_{s y}^{2}}{2 d_{p z} \cdot\left(d_{r w x}+0.5 h_{0}\right)}
$$

式中, $h_{0}$ 为相邻杀伤区火力衔接部分在可能完成任 务线外的杀伤区纵深, 这里设 $h_{0}=d_{s y}+d_{p z}-d_{r w x}$, 那 么(2) 式转变为

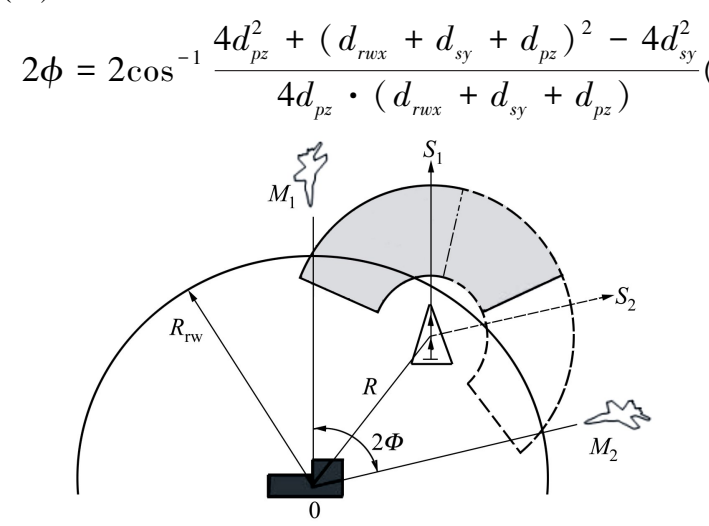

图 2 掩护角的物理意义

\section{2 相关概念关系分析}

1) 配置距离与水平杀伤区面积

鉴于地空反辐射混编群承担任务的性质, 这里 假定电子干扰机对混编群内常规防空武器的压制性 系数为 7 (雷达抗干扰能力为中等量级)。为避免地 空反辐射武器攻击, 空中干扰机一般在地空反辐射 武器杀伤区远界绕飞施放干扰, 因此, 地空反辐射武 器杀伤区远界 $D_{f f s s y}$ 、常规防空武器配置距离 $d_{i p z}$ 及干 扰机与常规防空武器制导雷达的距离 $R_{J}$ 满足以下 关系

$$
D_{f f s s y}=d_{i p z}+R_{J}
$$


因为 $D_{f f s s y}$ 是一个定值, 将 (4) 式代人 (1) 式, 经 转化常规防空武器配置距离 $d_{i p z}$ 与制导雷达最大搜 索距离 $R_{t}$ (目标与雷达的距离) 就形成了一一对应 关系即

$$
\frac{P_{j} G_{j}}{P_{t} G_{t}} \times \frac{4 \pi \gamma_{j}}{\sigma} \times \frac{G^{\prime}}{G_{t}} \times \frac{R_{t}^{4}}{\left(D_{f f s s y}-d_{i p z}\right)^{2}}=7
$$

依据经验制导雷达最大搜索距离与水平杀伤区 面积 $S$ 有以下函数关系

$$
S=k \pi\left(D_{\text {iwgrsy }}^{2}-D_{\text {iwgrsj }}^{2}\right) \frac{R_{t}^{2}}{D_{\text {iwgrss }}^{2}} q^{2}
$$

式中, $k, q$ 均为常数, $D_{\text {iwgrss }}, D_{\text {iwgrsy }}$ 分别为第 $i$ 个常规 防空武器无干扰条件下水平杀伤区近界和远界, $R_{t}$, $D_{\text {iwgrss }}$ 分别为有、无干扰条件下制导雷达的最大搜索 距离。

将(5) 式代人 (6) 式, 经转化得到杀伤区面积 与常规防空武器配置距离的函数关系式

$$
\begin{aligned}
S= & k q^{2} \pi\left(D_{\text {iwgrsy }}^{2}-D_{\text {iwgrsj }}^{2}\right) \cdot \\
& \frac{G_{t}\left(D_{f f s s y}-d_{i p z}\right)}{2 D_{i w g r s s}^{2}} \sqrt{7 P_{t} \sigma / \pi \gamma_{j} P_{j} G_{j} G_{t}^{\prime}}
\end{aligned}
$$

2) 配置距离与可能完成任务线

设第 $i$ 个常规防空武器配置距离 (与保卫目标 的距离) 为 $d_{i p z}$ 、杀伤区远界为 $D_{i s y}$, 为了能在空袭目 标到达可能完成任务线之前将其有效杀伤, 常规防 空武器配置位置距保卫目标距离与杀伤区远界的和 应大于空袭目标可能完成任务线的水平距离 $d_{r w x}$, 即 $d_{i p z}+D_{i s y}>d_{r w x}$ 。

\section{3 ) 责任扇区与掩护角}

责任扇区是地空反辐射混编群承担防空任务方 向的界定, 在水平面上为一个扇面角 $\theta$, 在此扇面角 范围内的所有空袭目标由地空反辐射混编群拦截射 击。混编群内常规防空武器若要实现以不低于指定 概率对责任扇区内来袭目标进行拦截, 须满足常规 防空武器对地面保卫目标掩护角之和不小于地空反 辐射混编群防空责任扇区即: $\sum_{i=1}^{n} 2 \phi_{i} \geqslant \theta$, 为确保防 空火力资源的有效性, 这里设定 $\sum_{i=1}^{n} 2 \phi_{i}=\theta$, 且地空 反辐射导弹武器系统工作扇区可覆盖混编群防空责 任扇区。

\section{4) 叠加杀伤区面积与综合作战效能}

定性来说, 在地空反辐射导弹武器系统掩护下, 常规防空武器若要实现对来袭目标杀伤效能最大 化, 需令叠加后的常规防空武器杀伤区面积最大。
这里设定常规防空武器杀伤区火力衔接时, 水平杀 伤区重叠部分面积占单个常规防空武器杀伤区面积 的 $5.2 \%$ (经验值)。

随着常规防空武器配置前伸, 其被干扰强度逐 渐增强, 对空袭目标最大搜索距离逐步减小, 水平杀 伤区面积逐渐压缩, 导致混编群综合杀伤效能不断 下降。如何在满足相关限制条件的同时, 令地空反 辐射混编群内常规防空武器的水平杀伤区面积最 大, 就成为该问题的求解目标。该问题也就转变为 多参数、多条件下的决策优化问题 ${ }^{[6-10]}$ 。

\section{2 兵力配置优化模型}

为建立地空反辐射混编群兵力配置优化数学模 型, 作如下简化想定: 某地空反辐射混编群配属 1 套 SA-ARM 武器系统、3 套同类型常规防空武器, 用以 承担某要地指定方向的对空防御任务,责任扇区扇 面角为 $100^{\circ}, \mathrm{SA}-\mathrm{ARM}$ 武器系统工作扇区可覆盖责 任扇区, 敌对方空袭编队在电子干扰飞机支援下实 施突防 (击)。在 SA-ARM 阵地位置已定的情况下, 3 套常规防空武器如何配置可使作战群对空防御作 战效能最大,如图 3 所示。将问题置于以地空反辐 射阵地为原点的平面直角坐标系内进行讨论, 第 $i$ 套防空武器的配置位置为 $\left(x_{i}, y_{i}\right)$, 则其配置距离为 $d_{i p z}=\sqrt{x_{i}^{2}+y_{i}^{2}}$ 。根据上述分析地空反辐射混编群 兵力配置优化的目标函数为

$$
\begin{gathered}
\max S=89.6 \% \times \sum_{i=1}^{3} k q^{2} \pi\left(D_{\text {iwgrsy }}^{2}-D_{\text {iwgrsj }}^{2}\right) \cdot \\
\frac{G_{t}\left(D_{f f s s y}-\sqrt{x_{i}^{2}+y_{i}^{2}}\right)}{2 D_{\text {iwgrss }}^{2}} \sqrt{7 P_{t} \sigma / \pi \gamma_{j} P_{j} G_{j} G^{\prime}}{ }_{t}
\end{gathered}
$$

同时,还应满足以下约束条件

$$
d_{i p z}+D_{i g r s y}>d_{r w x}, i=1,2,3
$$

$$
\begin{gathered}
\sum_{i=1}^{3} \cos ^{-1} \frac{4 d_{i p z}^{2}+\left(d_{r u x}+D_{i g r s y}+d_{i p z}\right)^{2}-4 D_{i g r s y}^{2}}{4 d_{i p z} \cdot\left(d_{r u x}+D_{i g r s y}+d_{i p z}\right)}=50 \\
i=1,2,3
\end{gathered}
$$

$\theta_{i} \neq \theta_{i+1} \neq \theta_{i+2} \neq \cdots \theta_{i+n}, i=1,2,3$ ( 常规装备配置点 方位角互不相等)

因为

$$
D_{i g r s y}=q^{2} D_{i w g r s y} \frac{G_{t}\left(D_{f f s s y}-d_{i p z}\right) \sqrt{7 P_{t} \sigma}}{2 D_{i w g r s s}^{2} \sqrt{\pi \gamma_{j} P_{j} G_{j} G^{\prime}{ }_{t}}}
$$


变为关于配置距离 $d_{i p z}$ 的 2 个约束。

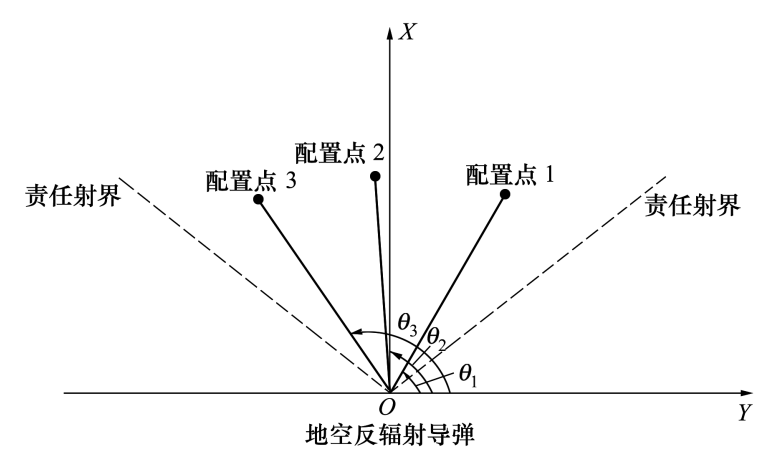

图 3 常规防空武器兵力配置示意图

\section{3 量子遗传算法的改进}

量子进化算法利用量子理论中有关量子态的叠 加和纠缠等特性,通过量子并行计算来求解问题,其 计算特性和硬件基础使得量子进化算法拥有巨大的 潜力。文献 [17] 提出了具有细胞结构种群的去中 心化的量子粒子群算法。算法重新定义了每个子种 群搜索方式,通过将子种群最优个体与固定范围的 局部粒子进化的方式进行搜索。文献 [18]通过设 置一种自适应邻域变化的方法, 将克隆机制引人量 子进化算法中。文献 [19] 针对实时车间调度问题, 提出了启发式量子进化算法, 算法使用基于剪枝交 叉方法提高种群的多样性。上述文献主要的改进在 于通过改变算子搜索方式进而避免算法陷人局部最 优, 但是这些算法的计算复杂度较高, 理解和实现难 度相对较大。本文基于基本量子遗传算法, 提出了 自适应旋转门搜索方式。该方法依据每个个体在种 群中偏离最优个体的程度, 自适应生成旋转门, 在保 证种群多样性和避免搜索陷人局部最优的同时, 最 大化提高收玫速度, 且与其他文献相比, 本文方法计 算简单、复杂度低、易于实现 ${ }^{[11-19]}$, 这与地空反辐射 混编群防空作战规划高时效性与简构性要求是相契 合的 ${ }^{[3,6]}$ 。

\section{1 量子遗传算法简述}

在量子遗传算法中最重要的是量子编码和量子 门的引人。量子编码是将染色体用量子的态矢量表 示, 使一条染色体表达为多个态的叠加, 从而增加了 种群的多样性和丰富性, 使算法能够在较小的种群 规模下寻优; 而量子门的引人确保了种群的更新, 使 算法能够快速收敛。

\section{1) 量子比特编码}

在量子计算中量子比特是最小的信息存储单 元,一个量子比特可用 3 种状态来表示, 分别是 0 态 1 态及 0,1 之间的任意叠加态, 其状态表达式为

$$
|\phi\rangle=\alpha|0\rangle+\beta|1\rangle
$$

式中, $\alpha$ 和 $\beta$ (复数) 为量子比特对应的概率幅, $|0\rangle$ 的概率为 $\alpha^{2} 、|1\rangle$ 态的概率为 $\beta^{2}$, 且两者满足关 系式

$$
\alpha^{2}+\beta^{2}=1
$$

在 QGA 中用一对复数表达一个量子比特, 具有 $n$ 个量子比特的系统可用如下方式表示

$$
\left|\begin{array}{l|l|l|l|l}
\alpha_{1} & \alpha_{2} & \alpha_{3} \\
\beta_{1} & \beta_{2} & \beta_{3}
\end{array}\right| \cdots\left|\begin{array}{l}
\alpha_{n} \\
\beta_{n}
\end{array}\right|
$$

式中, $\alpha_{i}^{2}+\beta_{i}^{2}=1, i=1,2,3 \cdots n_{\circ}$ 用这种方法来定义 染色体, 可使一条染色体同时表达多个态的叠加, 从 而实现了用较小的种群规模表达更多信息的目 的。

\section{2）量子门}

量子门是进行量子比特的操作机构, 随着 QGA 的不断发展演进, 目前量子门也有多种类型。本文 采用量子旋转门实现染色体的调整。量子旋转门相 当于传统遗传算法中的变异, 其调整操作为

$$
\left[\begin{array}{c}
\alpha_{i}^{\prime} \\
\beta_{i}^{\prime}
\end{array}\right]=\left[\begin{array}{cc}
\cos \theta_{i} & -\sin \theta_{i} \\
\sin \theta_{i} & \cos \theta_{i}
\end{array}\right]\left[\begin{array}{l}
\alpha_{i} \\
\beta_{i}
\end{array}\right]
$$

式中, $\theta_{i}$ 为旋转门的旋转角, 其大小和方向取决于 选定的调整策略, $\theta_{i}$ 过大则染色体更新幅度增加, 易 导致未全局寻优即收敛, $\theta_{i}$ 过小则染色体更新幅度 减小, 易导致局部寻优。由此可见, 旋转角的取值策 略对问题求解具有重要影响。

\section{2 算法的改进}

为实现算法的改进,此处引人自适应调整量子 旋转门旋转角的思想, 在种群保留和更新之间寻找 一个平衡点, 以避免早熟和局部寻优情况的出现。 基本考虑是, 在染色体距当前最佳适应度值较远时, 适当增大旋转角, 以提高染色体更新幅度, 强化全局 寻优能力; 在染色体距当前最佳适应度值较近时, 适 当减小旋转角, 以提高算法收玫速度。具体方法为: 以当前目标函数值对最优值之比为旋转角增减率的 依据, 确定旋转角基准值后旋转角随之确定, 即

$$
\theta_{i}=\theta_{m}\left(1-\frac{f}{f_{\text {max }}}\right)
$$

式中, $\theta_{m}$ 为旋转角基准值 $f$ 为当前目标函数值 $f_{\text {max }}$ 为每次计算的最优目标函数值。 


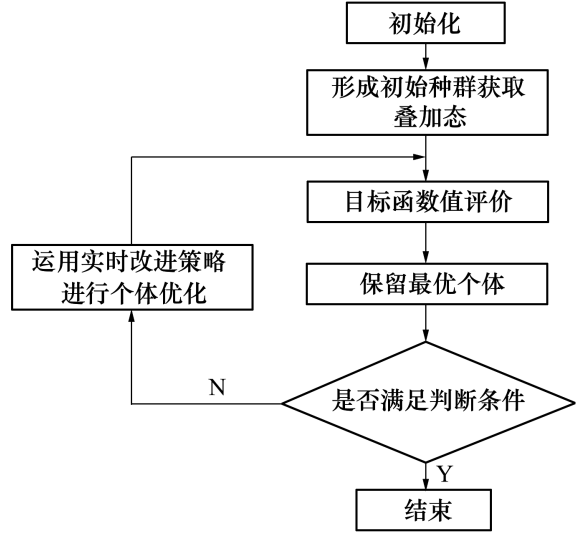

图 4 改进量子遗传算法流程

旋转角取值范围一般在 $0.001 \sim 0.1 \pi$ 之间 ${ }^{[11]}$, 鉴于地空反辐射混编群所属装备射程范围广、兵力
配置位置可选范围大, 为强化算法的全局寻优能力 及收玫速度, 需适当加大旋转角步进值, 因此这里 $\theta_{m}$ 取最大值 $0.1 \pi$, 改进量子遗传算法流程如图 4 所示。

\section{4 实例分析}

现设置作战场景典型参数,运用改进的量子遗 传算法对问题求解,并对结果进行分析。

\section{1 场景设定}

为更好地说明问题设置想定实例, 假设 $D_{f f s y}$ 为 $410 \mathrm{~km}, \sigma$ 为 $2 \mathrm{~m}^{2}, \gamma_{j}$ 为 $0.5, d_{r w x}$ 为 $140 \mathrm{~km}, D_{\text {iwgrss }}$ 为 $150 \mathrm{~km}, D_{\text {iwgrsy }}, D_{\text {iwgrs }}$ 分别为 $120,4 \mathrm{~km}, G_{t}, G_{t}^{\prime}, G_{j}$ 分 别为 $35,15,13 \mathrm{~dB}, P_{j}, P_{t}$ 分别为 1 和 $100 \mathrm{~kW}, 2$ 个常 数 $k=0.88, q=1.12$, 将以上数值代人后得目标函数 与约束条件如下

$$
\left\{\begin{array}{l}
\max S(c)=91131-74.1 \cdot \sum_{i=1}^{3} \sqrt{x_{i}^{2}+y_{i}^{2}} \\
G_{1}(c)=\max \left(16.7-0.37 \sqrt{x_{i}^{2}+y_{i}^{2}}, 0\right) \\
G_{2}(c)=\max \left(\sqrt{x_{i}^{2}+y_{i}^{2}}-\sqrt{3839.33+146.674 \sqrt{x_{i}^{2}+y_{i}^{2}}+0.0693\left(x_{i}^{2}+y_{i}^{2}\right)}, 0\right) \\
G_{3}(c)=\max \left(\left|\sum_{i=1}^{3} \cos ^{-1} \frac{4.2772 \cdot\left(x_{i}^{2}+y_{i}^{2}\right)+586.696 \cdot \sqrt{x_{i}^{2}+y_{i}^{2}}+15357.32}{986.4 \cdot \sqrt{x_{i}^{2}+y_{i}^{2}}+2.96 \cdot\left(x_{i}^{2}+y_{i}^{2}\right)}\right|-50,0\right) \\
\theta_{1} \neq \theta_{2} \neq \theta_{3}
\end{array}\right.
$$

在约束条件中, 对于 2 个不等式约束 $\left(G_{1}\right.$ 和 $\left.G_{2}\right)$ 和一个不等式约束 $\left(G_{3}\right)$, 采用罚函数的方法, 将 约束条件与目标函数一并处理, 对于不等式约束 $\theta_{1}$ $\neq \theta_{2} \neq \theta_{3}$ 则是将不满足约束条件的个体剔除。

本文的问题为最大值求解问题, 鉴于目标函数 的数值范围与约束条件的数值范围差异较大, 因此 采用自适应罚函数控制方法进行求解。

$$
\text { Fit }(c)=S_{\text {norm }}(c)+\lambda(t) \sum_{i=1}^{m} G_{i}(c)
$$

式中,惩罚系数自适应更新如下：

$$
\lambda(t+1)=\left\{\begin{array}{lc}
\left(1 / \eta_{1}\right) \lambda(t), \quad \text { if case \#1 } \\
\eta_{2} \lambda(t), \quad \text { if case \#2 } \\
\lambda(t), \quad \text { otherwise }
\end{array}\right.
$$

式中, $\lambda(t)$ 表示第 $t$ 代的罚函数, $\eta_{1}$ 和 $\eta_{2}$ 分别表示 迭代过程中惩罚系数变化参数, case \#1 表示在过去 $\mathrm{g}$ 代中, 最优个体均为不可行解, case \#2 表示在过去 $g$ 代中最优解为可行解。其原理可理解为: 若在此 之前所找到的最好个体均为可行解, 则表明惩罚系 数已足够大, 可适当减少来降低对不可行解的惩罚 压力; 若此前所找到的最好个体均为不可行解,则表 明惩罚系数过小,需适当增大来增强对不可行解的 惩罚力度。在实例求解中, $\eta_{1}$ 和 $\eta_{2}$ 分别设为 3 和 2 , $g$ 设为 5 。

\section{2 问题求解及结果分析}

为了对兵力配置优化模型进行验证, 并开展实 验结果分析, 分别采用本文提出的改进量子算法、基 本量子算法、粒子群算法及量子浴流算法对问题进 行计算。其中, 粒子群算法的加速因子 $c_{1}$ 和 $c_{2}$ 设为 $2, w$ 取值 0.729 ,量子浴流算法和基本的量子算法除 了种群规模没有额外的参数, 本文算法的主要参数 是 $\theta_{m}$ 设为 0.1 。每种算法在算例中种群规模 $N$ 设为 100 ,迭代终止次数设为 100,4 种算法第一次运行时 
最优解在迭代过程中的变化过程如图 5 所示。

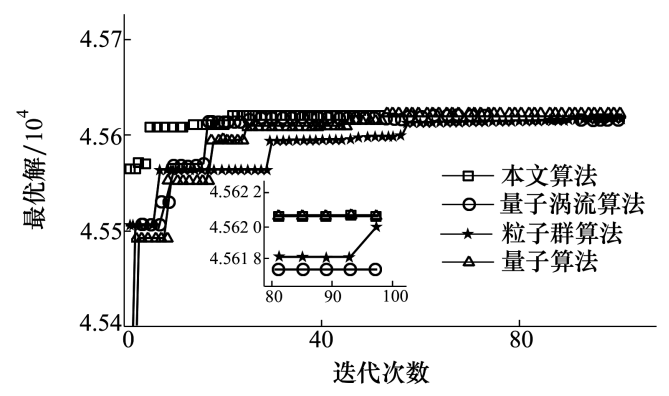

图 5 最优解与迭代次数关系图

表 1 为第一次运行得到的对应最佳目标函数值 的常规防空装备配置位置, 对照地空反辐射混编群
作战部署要求 ${ }^{[3]}$, 随机遴选若干组配置位置进行验 证, 定性对比结果证明了模型及算法在地空反辐射 混编群兵力配置问题求解方面的有效性。图 5 中, 从算法搜索最优解的趋势可以看出, 本文算法在前 期能够搜索到精度相对较高的最优解, 而其他算法 的搜索能力较差。尽管量子浴流算法能够很快搜索 出与本文算法精度相似的最优解, 但是最后的局部 搜索结果中,本文算法要优于量子涡流算法。为了 进一步验证算法的优越性,4种算法在案例中独立 运行 50 次, 计算最优值的均值和方差, 结果如表 2 所示。从表 2 可以看出, 本文的算法在收玫精度和 稳定性方面要优于其他 3 种算法。这充分体现了改 进 QGA 算法的优越性。

表 1 地空反辐射混编群常规装备配置位置

\begin{tabular}{ccccccc}
\hline \multirow{7}{*}{ 序号 } & \multicolumn{7}{c}{ 位置 } \\
\cline { 2 - 7 } & $x_{1}$ & $y_{1}$ & $x_{2}$ & $y_{2}$ & $x_{3}$ & $y_{3}$ \\
\hline 1 & 63.0403 & 144.8887 & -117.2230 & 106.0792 & 0.230816 & 158.1340 \\
2 & -120.8170 & 101.9162 & 112.8859 & 110.8357 & -49.0015 & 150.3214 \\
3 & 132.0424 & 87.0341 & 61.4385 & 145.6662 & -150.2570 & 49.1358 \\
4 & 102.4713 & 120.3341 & -99.0385 & 123.2336 & -12.0013 & 157.6980 \\
5 & 148.8276 & 53.3498 & -26.2317 & 155.9287 & 93.4682 & 127.5613 \\
$\ldots$ & $\ldots$ & $\ldots$ & $\ldots$ & $\ldots$ & $\ldots$ & $\ldots$ \\
48 & -8.42769 & 157.8172 & 118.9466 & 104.2514 & -78.1320 & 137.4387 \\
49 & -93.1527 & 127.7947 & -140.5200 & 72.51239 & 55.4492 & 148.0099 \\
\hline
\end{tabular}

表 2 目标函数值对比

\begin{tabular}{ccc}
\hline 算法类型 & 均值 & 方差 \\
\hline 本文算法 & 45620.68 & $2.68 \times 10^{-7}$ \\
量子涡流算法 & 45620.67 & $1.09 \times 10^{-5}$ \\
量子算法 & 45617.65 & 9.502988 \\
粒子群算法 & 45617.45 & 6.355908 \\
\hline
\end{tabular}

表 3 运行时间对比

\begin{tabular}{ccc}
\hline 算法 & 均值 & 方差 \\
\hline 本文算法 & 0.1612 & 0.000509 \\
量子浴流算法 & 0.153933 & $6.19 \times 10^{-5}$ \\
量子算法 & 0.0416 & $6.03 \times 10^{-5}$ \\
粒子群算法 & 0.043733 & 0.000252 \\
\hline
\end{tabular}

由表 3 可看出,本文算法在运行时间上均劣于 其他 3 种算法,运行 50 次,其周期均值达 $0.1612 \mathrm{~s}$, 考虑到兵力配置往往在战前开展, 因此对问题分析 而言该运行时间可控,能够满足实际需求。

\section{5 结 论}

量子遗传算法以其先进的结构设计和运行模 式,自提出伊始就展现了其在多目标多条件问题求 解中的独特优势。本文构建了地空反辐射混编群兵 力配置优化模型, 并结合混编群兵力配置特点, 对 QGA 旋转门旋转角设置进行了自适应改进, 经开展 简化实例 (同型号常规防空装备、等杀伤概率杀伤 目标) 计算及对比分析,证明了混编群兵力配置优 化模型的有效性及改进 QGA 在兵力配置优化问题 求解中的优越性, 计算结果可直接应用于兵力配置 
实践。结合问题实际, 后续可在量子比特编码、异型 常规防空装备目标分配、自卫式干扰目标拦截等环
节上进行改进完善,如引人量子灾变等思路,进一步 强化算法的全局寻优能力。

\section{参考文献:}

[1] 顾尔顺. 地空反辐射导弹综合分析研究 [J]. 地面防空武器, 2010(1) : 19-22

GU Ershun. Surface to Air Anti-Radiation Missile Analysis and Research[J]. Surface to Air Weapon,2010(1):19-22 (in Chinese)

[2] 刘屹立. 地空反辐射导弹武器系统的防空作用分析 [J]. 现代制造技术与装备,2016(3): 152-154

LIU Yili. Analysis of Surface to Air Anti-Radiation Missile System's Effect $[\mathrm{J}]$. Recent Manufacture Technology and Equipment, 2016(3) : 152-154 (in Chinese)

[3] 季军亮,段立波,田海林. 地空反辐射导弹混成群兵力部署研究 [J]. 飞航导弹,2017(1) : 49-54

JI Junliang, DUAN Libo, TIAN Hailin. Research on Surface to Air Anti-Radiation Hybrid Group Force Deploy[J]. CruiseMissile, 2017(1) : 49-54 (in Chinese)

[4] HKH K J H. Genetic Quantum Algorithm and its Application to Combinatorial Optimization Problem[C] // Proc of IEEE Congress on Evolutionary Computation, 2000: 1354-1360

[5] 夏辉. 美军舰载电子战飞机综述 $[J]$. 电子信息对抗技术, 2014(6):19-22

XIA Hui. Review of the USA Navy Carrier-Borne EW Aircraft $[\mathrm{J}]$. Electronic Information Warfare Technology, 2014(6) : 19-22 (in Chinese)

[6] 陈鸿猷, 郭有全, 王颖龙. 中国人民解放军空军地空导弹兵战术学 [M]. 北京: 解放军出版社, 2015

CHEN Hongyou, GUO Youquan, WANG Yinglong. Air Force Surface to Air Missile Army Tactics [M]. Beijing, PLA Publishing House, 2015 (in Chinese)

７］张永顺,童宁宁,赵国庆. 雷达电子战原理 [M]. 北京:国防工业出版社,2016

ZHANG Yongshun, TONG Ningning, ZHAO Guoqing. Radar Electronic Warefare Theory[ M]. Beijing, National Defence Industrial Engineering Publishing House, 2015 (in Chinese)

[8] 陶欢,周小平,孙永全,等. 一种基于干信比的电子干扰信号环境度量方法 $[\mathrm{J}]$. 太赫兹科学与电子信息学报.2017 (1): 59-64

TAO Huan, ZHOU Xiaoping, SUN Yongquan, et al. An Evaluation Method of Electronic Jamming Signal Environment Based on $\operatorname{SJR}[J]$. Journal of Terahertz Science and Electronic Information Technology, 2017(1) : 59-64 (in Chinese)

[9] 卢盈齐,李宁,陈刚. 反导部署的掩护角分析 [J]. 火力与指挥控制,2015(7): 85-88

LU Yingqi, LI Ning, CHEN Gang. Covering Angle Analysis of Anti-Missile Deployment [J]. Fire \& Command and Control, 2015(7): 85-88 (in Chinese)

[10] 寇占霖. 地空导弹射击理论及其应用 $[M]$. 西安: 空军工程大学, 2014

KOU Zhanlin. Shoot Theory and Application of Surface to Air Missile[M]. Xi'an, Air Force Engineering University, 2014 (in Chinese)

[11] 白小宝. 量子遗传算法研究 $[\mathrm{J}]$. 电脑开发与应用, 2013(1):9-15

BAI Xiaobao. Reaearch on Quantum Genetic Algorithm[J]. Exploitation and Applitation of Computers, 2013(1) : 9-15 (in Chinese)

[12] 蒋林利. 量子遗传算法研究现状综述 $[\mathrm{J}]$. 广西科技师范学院学报,2016(2): 130-133

JIANG Linli. Summarization of Quantum Genetic Algorithm Research Actuality [J]. Journal of Guangxi Science \& Technology Normal University, 2016(2):130-133 (in Chinese)

[13] 梁昌勇,柏华,蔡美菊,等. 量子遗传算法研究进展 [J]. 计算机应用研究,2012(7):2401-2405

LIANG Changyong, BAI Hua, CAI Meiju, et al. Advances in Quantum Genetic Algorithm[J]. Application Research of Computers, 2012(7): 2401-2405 (in Chinese)

[14] 杨荣. 基于量子遗传算法的物流业与制造业共生阶段分析 $[\mathrm{J}]$. 西北工业大学学报,2017，35(2):69-73

YANG Ying. Analysis of Logistics \& Manufacture Intergrowth Stage based on Quantum Genetic Algrithm[J]. Journal of Northwestern Polytechnical University, 2017, 35(2): 69-73 (in Chinese) 
[15] 于王乐,汪家权. 改进的 QGA-BP 模型在复杂水质预测中的应用 [J]. 模式识别与人工智能,2012(4): 705-708

YU Wangle, WANG Jiaquan. Application Research on Complex Water Quality Prediction with Improved QGA-BP Model[J]. PR \& AI, 2012(4):705-708 (in Chinese)

[16] 马永杰,云文霞. 遗传算法研究进展 $[J]$. 计算机应用研究, 2016(4) : 1201-1206

MA Yongjie, YUN Wenxin. Research Progress of Genetic Algorithm $[\mathrm{J}]$. Application Research of Computers, 2012(4) : 12011206 (in Chinese)

[17] FANG W, SUN J, CHEN H, et al. A Decentralized Quantum-Inspired Particle Swarm Optimization Algorithm with Cellular Structured Population $[\mathrm{J}]$. Information Sciences, 2016, 330:19-48

[18] LIU J, WANG H, SUN Y, et al. Adaptive Niche Quantum-Inspired Immune Clonal Algorithm[J]. Natural Computing, 2016, 15 (2) : 297-305

[19] KONAR D, BHATTACHARYYA S, SHARMA K, et al. An Improved Hybrid Quantum-Inspired Genetic Algorithm( HQIGA) for Scheduling of Real-Time Task in Multiprocessor System[J]. Applied Soft Computing, 2017, 53: 296-307

\title{
Application of Improved Quantum Genetic Algorithm in Optimization for Surface to Air Anti-Radiation Hybrid Group Force Deployment
}

\author{
JI Junliang ${ }^{1,2}$, WANG Minle ${ }^{2}$, SHANG Chang' ${ }^{\prime}{ }^{1}{ }$, GAO Jiale ${ }^{1}$ \\ $\left.\begin{array}{l}\text { 1.Air and Missile Defense College, Air Force Engineering University, Xi' an 710051, China; } \\ \text { 2.Rocket Force University of Engineering } \mathrm{Xi}^{\prime} \text { an } 710025 \text {, China }\end{array}\right)$
}

Abstract: In this paper, the concept and force deployment needs for anti-electronic jamming and defensing air of surface to air anti-radiation hybrid group was presented, the relationship between shield angle, deployment distance and effective electronic interference etc, were analyzed in the background of air raid battle which is with electronic support, force deployment optimization model of surface to air anti-radiation hybrid group was built based on the kill zone target function. In terms of the characteristic of hybrid group force deployment, quantum genetic algorithm ( QGA) was improved with self-adaption rotation angle, the problem which was based on a living example was solved with improved QGA. By contrast, the improved QGA is better in the respects of global optimization, rate of convergence and stability than QGA, particle swarm optimization algorithm and quantum vortex algorithm in the problem of optimization for surface to air anti-radiation hybrid group force deployment.

Keywords : quantum genetic algorithm; surface to air ati-radiation; hybrid group; force deployment; Q-gate 